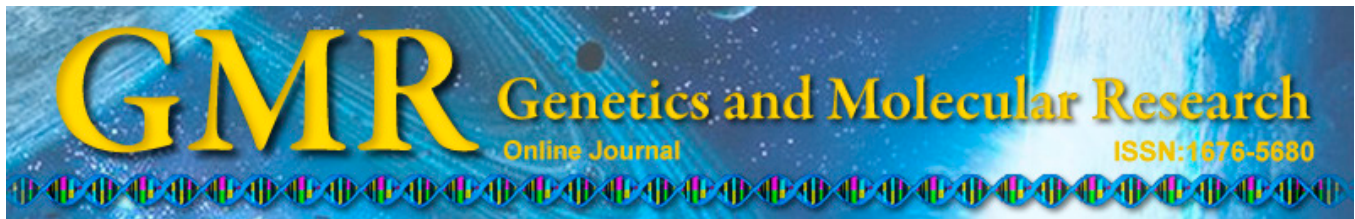

\title{
Effect of different time phases of radionuclide hepatobiliary scintigraphy on the differential diagnosis of congenital biliary atresia
}

\author{
Y.X. Guan', Q. Chen ${ }^{2}$, S.H. Wan' ${ }^{2}$ J.S. Huang ${ }^{2}$, X.Q. Yang ${ }^{1}$, L.J. Pan ${ }^{1}$, \\ Q.I. Zhang', Q. Zhang, ${ }^{1}$,.J. Ou ${ }^{1}$, X.W. Peng' ${ }^{2}$, S.Z. Liu' ${ }^{1}$, Q.J. Chen ${ }^{1}$ and J. Lou ${ }^{1}$ \\ ${ }^{1}$ The First Affiliated Hospital of Nanchang University, Nanchang, China \\ ${ }^{2}$ Children's Hospital of Jiangxi Province, Jiangxi, China \\ Corresponding author: Y.X. Guan \\ E-mail: yanxingguan2000@aliyun.com
}

Genet. Mol. Res. 14 (2): 3862-3868 (2015)

Received August 1, 2014

Accepted December 4, 2014

Published April 22, 2015

DOI http://dx.doi.org/10.4238/2015.April.22.15

\begin{abstract}
To investigate the value and essentiality of 6- and 24-h delay hepatobiliary scintigraphy in the differential diagnosis of biliary atresia (BA), we retrospectively analyzed 197 infants (121 boys/76 girls; age range, 3-205 days; average age, 63.9 days) admitted to Jiangxi Children's Hospital for persistent jaundice ( $>2$ weeks), hepatosplenomegaly, and abnormal liver function. After receiving anti-inflammatory treatment and cholagogic pre-treatment for 7-10 days without a clear diagnosis, the children underwent $99 \mathrm{mTc}$-labeled diethylacetanilide-iminodiacetic acid hepatobiliary scintigraphy. BA and infant hepatitis syndrome were diagnosed in 107 and 90 infants, respectively after laparoscopic cholangiography, surgical pathology, or 6-month clinical follow-up. The diagnostic efficiencies of hepatobiliary scintigraphy for BA were evaluated within 50 min and at 6 and $24 \mathrm{~h}$. The areas under the receiver operating characteristic curves within $50 \mathrm{~min}$, at 6 and $24 \mathrm{~h}$ were $0.696,0.829$, and 0.779 , suggesting poor diagnostic value within $50 \mathrm{~min}$, but improvement at 6 and $24 \mathrm{~h}$. The compliance rate of 6- and 24-h imaging for BA diagnosis was 89.34\% (176/197;
\end{abstract}


paired chi-square test Kappa value, 0.77; P > 0.05), signifying high consistency. The diagnostic efficiency values of 6-/24-h imaging for BA diagnosis were sensitivity (90.65/89.72\%), specificity (74.44/78.89\%), accuracy $(83.25 / 84.77 \%)$, positive and negative predictive values (80.83/83.48\% and 87.01/86.59\%), with no significant difference $(\mathrm{P}>$ 0.05). To provide optimal treatment in early BA, the- 6-h hepatobiliary scintigraphy had practical value, especially when combined with tomographic or dynamic imaging; 24-h delay imaging was deemed unnecessary because it was not significantly superior.

Key words: Hepatobiliary scintigraphy; Biliary atresia; Infant hepatitis syndrome

\section{INTRODUCTION}

Although biliary atresia (BA) and infant hepatitis syndrome (IHS) are 2 major causes of infant persistent jaundice, the treatment and prognosis for the 2 conditions are quite different. Approximately $30 \%$ of IHS patients will progress to cirrhosis if they do not receive symptomatic treatment. Meanwhile, BA requires immediate surgery or the patients advance to liver failure and death (Chen and Zhuang, 2013). Thus, a non-invasive method that is also fast, simple, and practical has great clinical value in the differential diagnosis of BA.

A commonly used non-invasive method for BA diagnosis is $99 \mathrm{mTc}$-labeled diethylacetanilide-iminodiacetic acid (99mTc-EHIDA) hepatobiliary scintigraphy. However, the routine use of 24-h delay imaging is tedious and time consuming, and several studies have reported its negative influence on the diagnostic accuracy (Guo et al., 2012). In this study, we performed a retrospective analysis to investigate the diagnostic efficiency of different time phases of radionuclide hepatobiliary scintigraphy on BA diagnosis to explore the possibility of reducing the imaging time to reach a diagnosis.

\section{MATERIAL AND METHODS}

\section{Clinical data}

We performed a retrospective analysis of 197 infants who were admitted to Jiangxi Children's Hospital from June 2010 to January 2013 because of persistent jaundice $(>2$ weeks), hepatosplenomegaly, and abnormal liver function. Among these infants, 121 were boys and 76 were girls, with ages ranging from 3 to 205 days (average age, 63.9 days). Infants were excluded if they had received a diagnosis of/ had undergone treatment for the following conditions: pre-hepatic jaundice, cytomegalovirus hepatitis, and congenital bile duct dilatation (Qiong, 2005). The blood bilirubin levels of the infants were measured and they were assessed by using $99 \mathrm{mTc}$-EHIDA hepatobiliary scintigraphy after they had undergone 7 days of antiinflammatory treatment with Transmetilor or a similar agent and cholagogic pre-treatment without receiving a clear diagnosis. After laparoscopic cholangiography, surgical pathology, or clinical follow-up for 6 months, 90 infants with blood bilirubin levels ranging from 74.6 to $386.2 \mu \mathrm{M}$ were ultimately diagnosed with IHS, while 107 with blood bilirubin levels ranging from 134.6 to $322.1 \mu \mathrm{M}$ (except for 1 case with $96.5 \mu \mathrm{M}$ ) were diagnosed with BA. 


\section{Method}

A S000045 Discovery VH dual probe single-photon emission computerized tomography (SPECT)/CT instrument with a low-energy, high-resolution parallel-hole collimator (General Electric Co., USA) was used. The infants were fasted for $4-6 \mathrm{~h}$ before the test, and they were administered $10 \%$ chloral hydrate solution $(4-10 \mathrm{mg} / \mathrm{kg}$ ) orally at $30 \mathrm{~min}$ before injection of the imaging agents. The infants were placed in a supine position, and the imaging field included the liver, intestine, and bladder. Planar radionuclide hepatobiliary imaging was performed immediately after IV injection of 99mTc-EHIDA, with a matrix of 64 x 64 pixels and magnification of 1.5; continuous dynamic acquisition was performed for 50 min with a velocity of 1 frame/min. Subsequent acquisition was performed with a matrix of 256 x 256 pixels and magnification of 1.5 at 6 and $24 \mathrm{~h}$ after injection; images were obtained in other positions to determine the radioactive image area, if necessary. The data were collected by using the counting method, and a decision was made by 2 skilled nuclear medicine physicians, separately. The first frame of dynamic acquisition was the start point (500-1000 k), and those without functional imaging of the gall bladder and/or bowel at 6 or $24 \mathrm{~h}$ were determined to be suspected BA (recorded as positive). In contrast, functional imaging of the gall bladder and/or bowel indicated a diagnosis of IHS (recorded as negative).

\section{Statistical analysis}

The SPSS17.0 statistical software [Norman H. Nie, C. Hadlai (Tex) Hull and Dale H. Bent in Stanford University of American] was used for data analysis. Receiver operating characteristic (ROC) curves of 50-min dynamic imaging, 6-h imaging, and 24-h imaging were drawn, and the area under the curve (AUC ${ }^{\mathrm{ROC}}$ ) was calculated to evaluate the diagnostic efficacy, with $0.50<\mathrm{AUC}^{\mathrm{ROC}} \leq 0.70$ indicating poor diagnostic value, $0.70<\mathrm{AUC}^{\mathrm{ROC}} \leq 0.90$ indicating middle diagnostic value, and $\mathrm{AUC}^{\mathrm{ROC}}>0.90$ indicating high diagnostic value. The consistency of the diagnosis at different time phases was evaluated by using Kappa analysis. A Kappa P value within 0.01-0.20 signifies low consistency, 0.21-0.40 signifies mild consistency, 0.41-0.60 indicates moderate consistency, 0.61-0.80 indicates high consistency, and 0.81-1.00 signifies complete consistency. The sensitivity, specificity, accuracy, and positive predictive value and negative predictive value were calculated based on the evaluation method of diagnostic efficacy.

\section{RESULTS}

\section{Diagnostic value of hepatobiliary scintigraphy within $50 \mathrm{~min}$ and at 6 and $24 \mathrm{~h}$}

The respective $\mathrm{AUC}^{\mathrm{ROC}}$ [95\% confidence interval $\left.(\mathrm{CI})\right]$ values of hepatobiliary scintigraphy within $50 \mathrm{~min}$ and at 6 and $24 \mathrm{~h}$ were 0.69 (0.62-0.773), $0.829(0.767-0.891)$, and $0.779(0.711-0.848)$. This suggested a poor diagnostic value within $50 \mathrm{~min}$, whereas this value greatly improved when the assessment was performed at 6 and $24 \mathrm{~h}$.

\section{Imaging results of hepatobiliary scintigraphy at 6 and $24 \mathrm{~h}$}

Among all 197 infants, 115 were determined as suspected BA (i.e., positive) in both 
time phases, while 61 were determined as IHS (i.e., negative) in both time phases. The compliance rate of the imaging results between the 2 phases was $89.34 \%(176 / 19)$, with Kappa $=$ 0.77 in the paired chi-square test $(\mathrm{P}>0.05)$, revealing high consistency between the 2 phases (Figure 1A-C, Figure 2A-C, Figure 3A-C, and Figure 4A-C). Among 107 infants ultimately diagnosed as BA, 97 were determined as suspected positives at $6 \mathrm{~h}$ and 96 were determined as suspected positives at $24 \mathrm{~h}$. Therefore, the sensitivities of 6- and 24-h imaging were $90.65 \%$ (97/107) and 89.72\% (96/107), respectively. Among the 90 infants who were ultimately diagnosed with IHS, 71 and 67 were determined as negative by 6-h imaging and by 24-h imaging, respectively. Thus, the specificities of imaging at 6 and $24 \mathrm{~h}$ were $78.89 \%(71 / 90)$ and $74.44 \%$ (67/90), respectively, and the respective false positive rates of the 2 phases were $21.11 \%$ $(19 / 90)$ and $25.56 \%(23 / 90)$. Based on the information above, the accuracies were $85.28 \%$ $(168 / 197)$ and $82.74 \%(163 / 197)$, the positive predictive values were $83.62 \%(97 / 116)$ and $80.67 \%(96 / 119)$, and the negative predictive values were $87.65 \%(71 / 81)$ and $85.90 \%(67 / 78)$ for imaging at 6 and $24 \mathrm{~h}$, respectively. Further, these aspects did not differ significantly between the 2 phases $(\mathrm{P}>0.05)$. The results are shown in Table 1 .
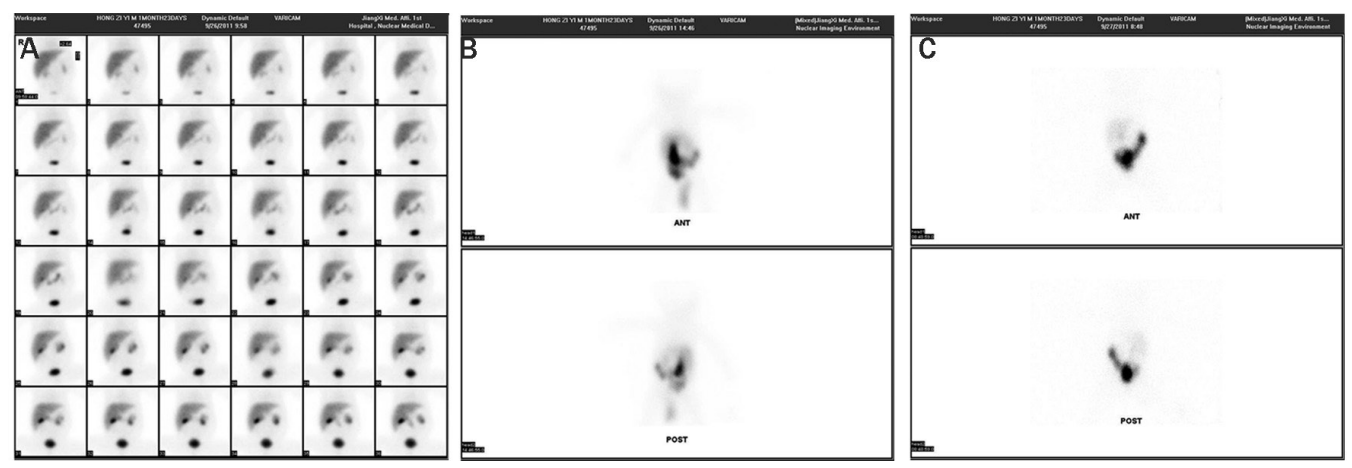

Figure 1. Negative hepatobiliary scintigraphy results at different time points (normal infants). (A) Gall bladder image within $50 \mathrm{~min}$. (B) Intestine image at $6 \mathrm{~h}$ [top panel, anterior (ANT); bottom panel, posterior (POST)] and (C) at $24 \mathrm{~h}$ (top panel, ANT; bottom panel, POST).
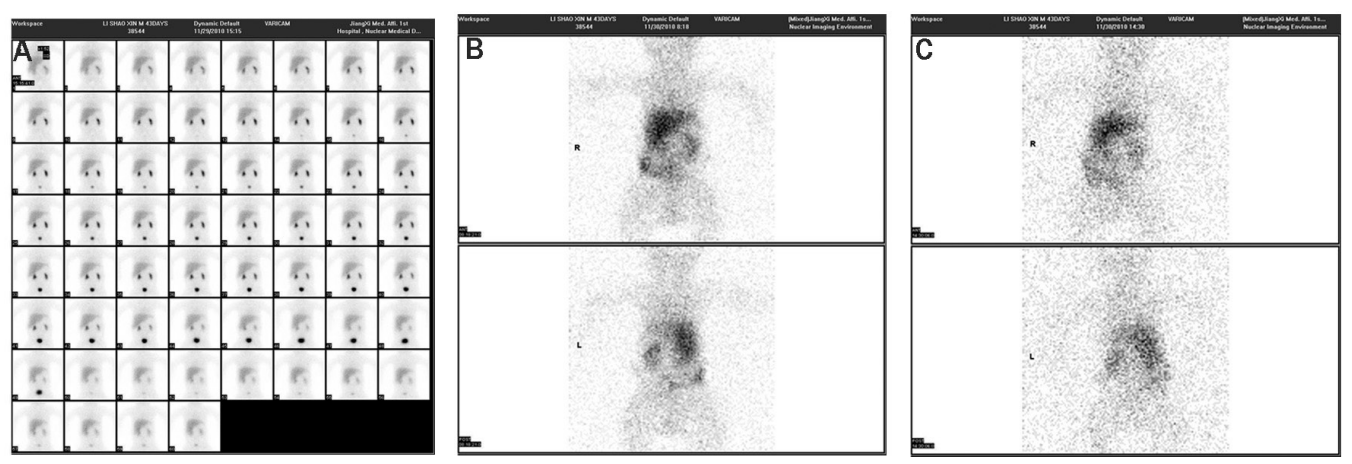

Figure 2. Negative hepatobiliary scintigraphy results at different time points (clinically diagnosed as IHS). (A) The gall bladder or intestine image was absent within $50 \mathrm{~min}$. (B) Intestine image at $6 \mathrm{~h}$ (top panel, ANT; bottom panel, POST) and (C) at $24 \mathrm{~h}$ (top panel, ANT; bottom panel, POST). 

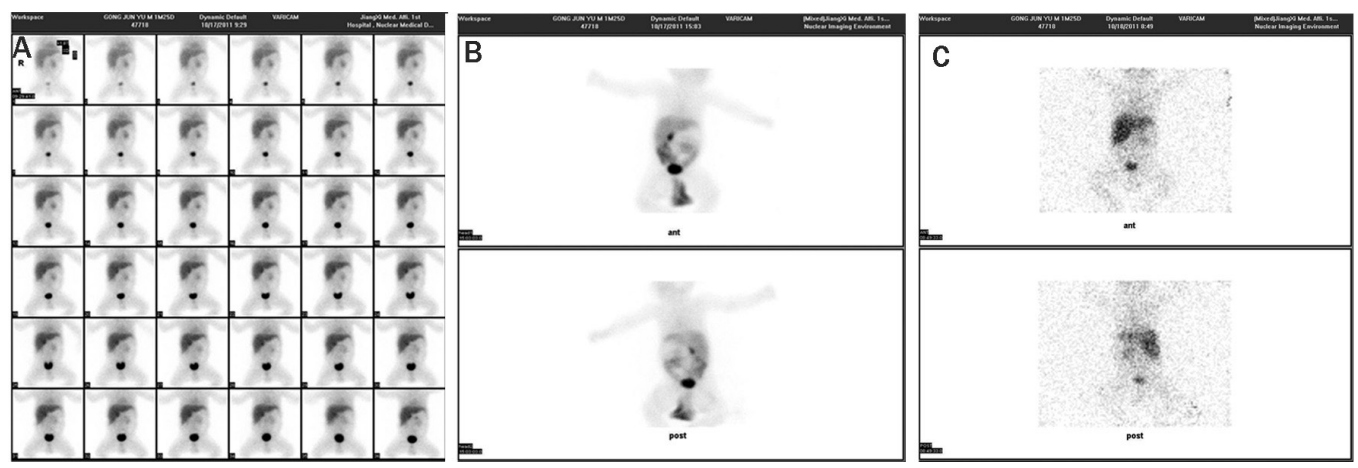

Figure 3. Inconsistency between the 6- and 24-h results (clinically diagnosed as IHS). (A) A gall bladder or intestine image was absent within $50 \mathrm{~min}$. (B) Intestine image at $6 \mathrm{~h}$ (top panel, ANT; bottom panel, POST). (C) The intestine image was absent at $24 \mathrm{~h}$ (top panel, ANT; bottom panel, POST).
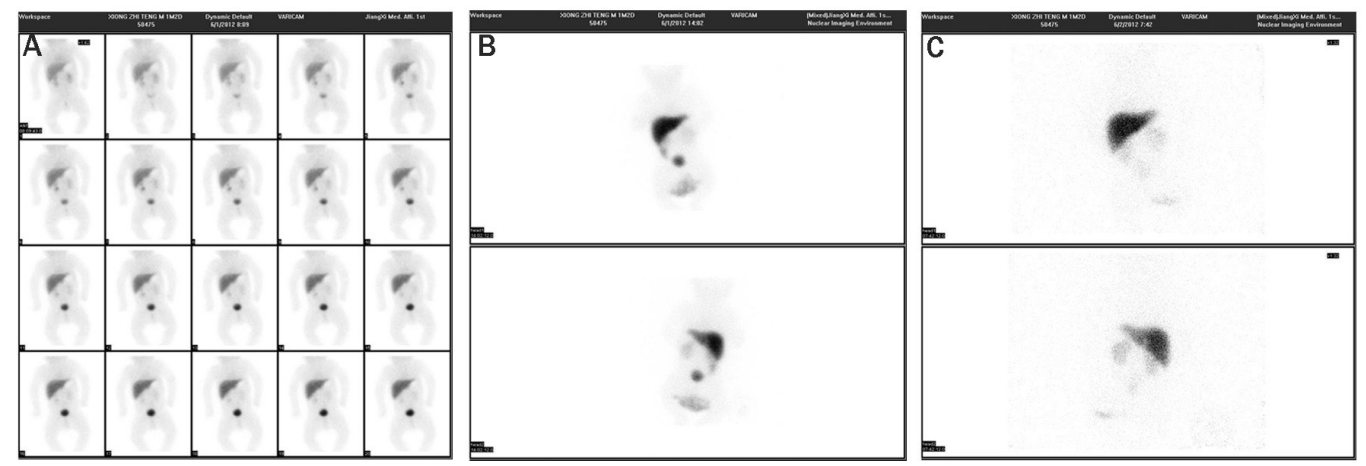

Figure 4. Positive hepatobiliary scintigraphy results at different time points (pathological diagnosis of BA). (A) The gall bladder or intestine was absent at $50 \mathrm{~min}$, (B) $6 \mathrm{~h}$ (top panel, ANT; bottom panel, POST, and (C) $24 \mathrm{~h}$ (top panel, ANT; bottom panel, POST).

Table 1. Diagnostic efficacy of 6- and 24-h imaging.

\begin{tabular}{lccccc}
\hline Imaging end point & Sensitivity & Specificity & Accuracy & Positive prognostic value & Negative prognostic value \\
\hline $6 \mathrm{~h}$ & $90.65 \%$ & $78.89 \%$ & $85.28 \%$ & $83.62 \%$ & $87.65 \%$ \\
$24 \mathrm{~h}$ & $89.72 \%$ & $74.44 \%$ & $82.74 \%$ & $80.67 \%$ & $85.90 \%$ \\
$\chi^{2}$ & 0.053 & 0.7058 & 0.6878 & 0.5921 & 0.3258 \\
$\mathrm{P}$ value & $>0.05$ & $>0.05$ & $>0.05$ & $>0.05$ & $>0.05$ \\
\hline
\end{tabular}

\section{DISCUSSION}

Delays in the diagnosis and treatment are crucial factors affecting the prognosis of BA and IHS. The clinical symptoms appear late or are not obvious in some BA infants, causing delayed diagnosis or even misdiagnosis. However, because a specific diagnostic method does not yet exist for BA, the treatment of liver protection and choleresis with routine examination is currently the clinical routine for infants with BA. The usefulness of the application of 
endoscopic retrograde cholangiopancreatography (ERCP), magnetic resonance cholangiopancreatography (MRCP), or CT is disputed, and these techniques are not recommended because of medical expense limits (Zheng, 2012). Radionuclide hepatobiliary scintigraphy is a noninvasive method that can evaluate the function of the hepatobiliary system and the patency of the biliary system by dynamic observation of the process in which a hepatobiliary-imaging agent is circulated like bilirubin via liver uptake, metabolism, and biliary excretion to the intestine. In patients with BA, visible imaging of the gall bladder or intestine is lacking because the imaging agent cannot be discharged into the intestine through the biliary system due to occlusion or absence of the bile duct. However, although hepatic inadequacy exists in infants with IHS, the extrahepatic bile ducts are clear; consequently, imaging of the gall bladder and intestine can generally be observed from 80 min to a maximum of $3 \mathrm{~h}$ (Zhang, 2008). Therefore, functional imaging of the gall bladder or intestine is enough to exclude the diagnosis of BA.

In clinical practice, hepatobiliary scintigraphy imaging at $24 \mathrm{~h}$ is usually thought to be necessary in order to increase the diagnostic accuracy. However, 24-h imaging requires repeated application of chloral hydrate, and the image quality is quite poor (Guo et al., 2012). On the other hand, pre-administration of anti-inflammation and cholagogic drugs has been reported to have a positive effect on dredging the biliary system and improving intestinal imaging, thereby improving the diagnostic efficacy (Shao and Han, 2009). In this study, we administered phenobarbital, phenobarbital with betamethasone, or ursodeoxycholic acid for 7-10 days before planar imaging, and we observed a favorable diagnostic effect in 6- and 24-h imaging. Moreover, the results for the 2 time phases were highly consistent, with no significant differences found in the diagnostic efficacies (consisting of the sensitivity, specificity, accuracy, and positive and negative prognostic values) of the 2 phases. This is presumably because the 6-h phase provided a larger amount of imaging information and better image quality. After pre-treatment, the biliary excretion function of infants with IHS was improved; hence, excretion of the imaging agent was accelerated, and obtaining adequate images at $6 \mathrm{~h}$ was possible. Meanwhile, due to decay of the $99 \mathrm{mTc}$ radiation, image information had decreased after $24 \mathrm{~h}$, so the small amount of injected imaging agent was difficult to discern when excretion remained low or had increased only slightly. Although the information provided by the image was increased by prolonging the imaging time to $24 \mathrm{~h}$, the resolution was not improved because of background amplification.

The images of the 90 infants with HIS in our study showed that the false positive rate was high for both 6- and 24-h imaging (21.11 and 25.56\%, respectively). However, in all 23 false-positive cases, the functional image of the gall bladder and/or intestine was more obvious in 6-h imaging than in 24-h imaging, and we propose several possible reasons for this observation. First, the high total blood bilirubin in these patients $(386.2 \mu \mathrm{M}$ in 1 case, $200-386.1 \mu \mathrm{M}$ in 5 cases, 108.8-200.4 $\mu \mathrm{M}$ in 16 cases, and $87.1 \mu \mathrm{M}$ in 1 case) resulted in competitive inhibition with 99mT-EHIDA for hepatocyte uptake; therefore, less imaging agent uptake resulted in a decrease in intestine imaging (Chen and Zhuang, 2003). Second, severe cholestasis caused a decrease in hepatocyte uptake of 99mT-EHIDA, also resulting in a decrease in intestine imaging. Third, the 24-h bowel imaging had quite low resolution, thereby making it difficult to interpret the image, despite the presence of some intestine imaging. In clinical practice, most infants with IHS who are misdiagnosed with BA are classified as the cholestasis type during laparotomy, which requires biliary drainage. Moreover, 24-h delay imaging did not effectively reduce the false-positive rate in cases with a high blood bilirubin level and severe liver damage. Therefore, the application of laparoscopic surgery as a treatment and diagnostic method has more practical value than that of 24-h delay imaging. 
The phenobarbital test is a classical method in hepatobiliary dynamic imaging for decreasing the false positive rate and elevating the specificity of BA diagnosis. However, we did not perform the phenobarbital test based on several considerations. First, the optimal treatment opportunity would have been more readily missed because the process would have been longer and more tedious, particularly when regular 24-h imaging did not show the gall bladder and intestine; therefore, a second phenobarbital test would have been needed. Second, a known side effect of phenobarbital is liver damage, and its application in liver disease is based on the condition of the patient (Zhang, 2006). In our study, most infants had a high blood bilirubin level and severe liver damage, and clinicians are reluctant to administer phenobarbital in such cases. By pre-treating the patients for 7-10 days with anti-inflammation and cholagogic medicine, we guaranteed the safety of the infants through opportune treatment and we improved the diagnostic efficacy of hepatobiliary scintigraphy. In addition, it has been reported that hepatobiliary planar imaging with $99 \mathrm{mTc}$-EHIDA plus tomography or 1-h hepatobiliary scintigraphy provides more radioactive imaging of the gall bladder or intestine than 24-h delay imaging (Shao et al., 2008; Yang et al., 2009; Xu et al., 2009). In the study by Sevilla et al. (2007), the diagnostic efficacy for BA was compared among plane imaging 4-6 $\mathrm{h}$ after imaging agent injection, planar imaging 4-6 $\mathrm{h}$ after imaging agent injection plus tomography, and tomography 4-6 h after phenobarbital injection, and the sensitivities of the 3 techniques were found to be identical. Moreover, the necessity and significance of 6-h hepatobiliary scintigraphy with 99mTc-EHIDA was emphasized when the specificity of plane imaging 4-6 h after imaging agent injection plus tomography was shown to be equivalent to, or even better than, the specificity reported for phenobarbital interventional in the literature.

In conclusion, given that the diagnostic efficacy of hepatobiliary scintigraphy with $99 \mathrm{mTc}-\mathrm{EHIDA}$ at $6 \mathrm{~h}$ was equal to that of 24-h delay imaging (after pre-treatment with antiinflammation and cholagogic medicine), we conclude that planar hepatobiliary scintigraphy at $6 \mathrm{~h}$ has practical value, especially when combined with tomography or dynamic imaging. Thus, we recommend that this technique be applied in order to provide the optimal treatment opportunity for infants with severe liver damage or long-term illness. In contrast, we propose that 24-h delay imaging is not necessary because it was not significantly superior to 6-h imaging.

\section{REFERENCES}

Chen Y and Zhuang HM (2013). Pediatric Nuclear Medicine. People's Health Publishing House, Beijing, May, 29-33. Guo Q, Yang J and Yang Y (2012). Delayed diagnosis of jaundice in children. J. Clin. Pediatr. Surg. 11: 53-55.

Qiong J (2005). Pediatric diagnostic procedures. Jiangsu Science and Technology Press, Nanjing, 180-184.

Shao GQ and Han JK (2009). Advances in imaging diagnosis and differential diagnosis of biliary atresia and infantile hepatitis syndrome. J. Med. Imag. 19770-19772.

Shao GQ, Han JK, Wang F and Li SH (2008). Differential diagnosis value of hepatobiliary section imaging and r-glutamyltransferase in persistent infantile jaundice. J. Appl. Clin. Pediatr. 23: 1510-1512.

Sevilla A, Howman-Giles R, Saleh H, Trpezanovski J, et al. (2007). Hepatobiliary scintigraphy with SPECT in infancy. Clin. Nucl. Med. 32: 16-23.

Xu XF, Liu YJ, Ma YH and Sheng DD (2009). 99mTc-EHIDA hepatobiliary scintigraphy combined with dynamic scan delay differential diagnosis of infant jaundice. Acta Univ. Med. Nanjing (Nat. Sci.) 29: 418-419.

Yang JG, Ma DQ, Li CL and Zou LF (2009). Significance of 99mTc-diethyl iminodiacetic acid hepatobiliary planar and tomographic scintigraphy in diagnosis on biliary atresia. J. Appl. Clin. Pediatr. 24: 1504-1505.

Zhang JZ (2008). Pediatric Abdominal Surgery. 1st edn. Zhejiang Science and Technology Press, Hangzhou, 421-422.

Zheng S (2012). Standardization of diagnosis and treatment of biliary atresia. J. Clin. Pediatr. Surg. 11: 241-241.

Zhang SJ (2006). Danshen combined treatment of hepatitis B and phenobarbital hyperbilirubinemia. Clin. J. Hepatol. 22 : 143-144. 